\title{
Formation of Twins and Stacking Faults during the Primary Recrystallization of Pure Nickel
}

\author{
By Pierre Merklen*, Ei-ichi Furubayashi** \\ and Hidehiko Yoshida**
}

\begin{abstract}
The formation of annealing twins has been examined by observing $96 \%$ rolled and electrolytically thinned high-purity nickel foils in a heating stage of a $500 \mathrm{kV}$ electron microscope.

The thickness dependence of the recrystallization temperature is found to be large; $500^{\circ} \mathrm{C}$ in specimens $0.2 \mu$ thick, $350^{\circ} \mathrm{C}$ in those $0.7 \mu$ thick as compared to $250^{\circ} \mathrm{C}$ in bulk ones. In specimens $0.2 \mu$ thick which are transparent for $100 \mathrm{kV}$ electrons, no annealing twins ars formed, whereas specimens $0.7 \mu$ thick can generate many twins similar to those in bulk-annealed specimens.

The nucleation sites of the annealing twins are always high-angle boundaries in motion, and the twins are divided into two classes from a morphorogical point of view; parallel-sided twins and thin twins, respectively. The parallel-sided twins result if the twinning plane is nearly parallel to the recrystallized grain boundary. These twins can grow to an extensive size through the migration of the high-angle boundaries, as in the case of usual recrystallized grain growth. On the other hand, the twinning planes of the thin twins are almost perpendicular to the boundary. The thin twins grow through a successive pile-up of stacking faults, probably by the pole mechanism, during dragging by the migrating boundary. The formation of twins is largely dependent on the local deformation texture at the site of twin nucleation.

A mechanism which conforms to the 'stimulation theory' proposed by Burgers has been considered to be the most plausible explanation of forming the parallel-sided twins.
\end{abstract}

(Received February 25, 1970)

\section{Introduction}

In order to investigate in detail the mechanism of recrystallization and grain growth, continuous observation on a microscopic scale is most useful. Several experimental works along this line have been tried with a $100 \mathrm{kV}$ electron microscope by heating the specimens inside the microscope $\mathrm{e}^{(1) \sim(3)}$. However, the thickness of the specimens observable with the $100 \mathrm{kV}$ electron microscope was too small for recrystallization to occur in the same way as in bulk specimens. For instance, in the case of high purity nickel rolled by $96 \%$, the recrystallization temperature was $500^{\circ} \mathrm{C}$ for the thin foils as against $250^{\circ} \mathrm{C}$ for the bulk specimens, and annealing twins which were reported to occur frequently in the bulk materials were never found in the foil-annealed thin specimens ${ }^{(3)}$.

Recently Fujita et al. showed that the high voltage electron microscope is very useful for the above mentioned purpose $^{(4)}$. Therefore, in the present work, a continuous observation on the recrystallization of pure nickel has been carried out using a $500 \mathrm{kV}$ electron microscope, with which one can observe specimens as thick as about $0.7 \mu$. In particular, the formation of twins and stacking faults during the primary recrystallization has been studied in detail.

It is inferred from the following two facts that the recrystallization behavior observed in the present work is

* Now at Pompey Steelworks Co., Nancy, France.

** Metal Physics Division, National Research Institute for Metals, Tokyo.

(1) J. E. Bailey : Phil. Mag., 5 (1960), 833.

(2) H. Hu : Trans. Met. Soc. AIME, 224 (1962), 75.

(3) P. Merklen : Thesis, C. N. R. S.,(1968).

(4) H. Fujita et al. : Japan. J. Appl. Phys., 6 (1967), 214 ; Trans. National Res. Inst. Metals, 9 (1967), 95. almost the same as that in bulk specimens. Firstly, the annealing twins were frequently formed as in bulk specimens, and secondly, the recrystallization temperature for the $96 \%$ rolled specimens was $350^{\circ} \mathrm{C}$ for the present case, which is much lower than that of thin foils $0.2 \mu$ thick $\left(500^{\circ} \mathrm{C}\right)$ though still higher than that of bulk specimens $\left(250^{\circ} \mathrm{C}\right)$.

\section{Experimental Procedure}

The specimens used were the same high-purity nicke' as that previously used by one of the authors ${ }^{(3)}$. The purity was estimated by the ratio of electrical resistivity $\left(\rho_{20.3} / \rho_{294} \sim 3 \times 10^{-3}\right)$ to be $25 \mathrm{ppm}$ of the total impurity content. Slices $5 \mathrm{~mm}$ thick were cut out from a rod of the as-melt polycrystalline specimen and rolled to a thickness of $0.2 \mathrm{~mm}$ ( $96 \%$ reduction) at room temperature. These rolled sheets were electrolytically thinned for the microscope observation in a solution of perchloric acid $20 \%$ and glacial acetic acid $80 \%$ of volume. The continuous observation was carried out with a $500 \mathrm{kV}$ electron microscope, Shimadzu SMH-5 B type, using a heating stage mounted on a universal goniometer ${ }^{(5)}$. The specimen thickness was determined by measuring the projected width of a (111) coherent twin boundary on the photographic plates and found to be $0.5 \sim 0.7 \mu$.

\section{Experimental Results}

\section{Parallel-sided twins}

Annealing twins in some $f c c$ metals and alloys which have been recrystallized after deformation are mostly of

(5) H. Fujita et al. : Trans. Nat. Res. Inst. Metals, $9(1967), 54$. 
the shape of parallel-sided lamellae ${ }^{(6) \sim(8)}$. In the present work on nickel parallel-sided twins were also frequently observed during the primary recrystallization. A series of photographs is Photo. 1 show an example of how annealing twins of this type are developed when heated
Since both the grains B and C are twin-related with the grain A through the same family of (111) twinning plane, they have an identical orientation. Thus a parallel-sided twin lamella was formed. Once the grain was bounded by the two parallel coherent twin planes, the grain $\mathrm{A}$

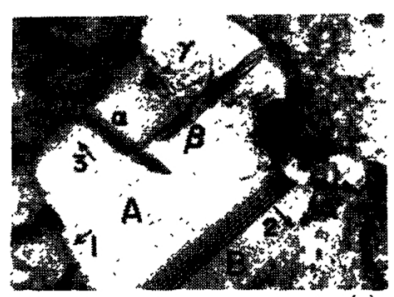

(a)

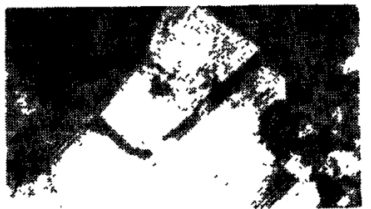

(b)

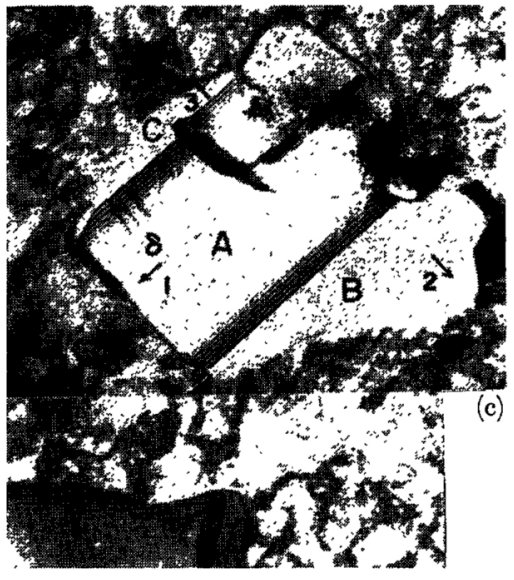

$1 \mu$
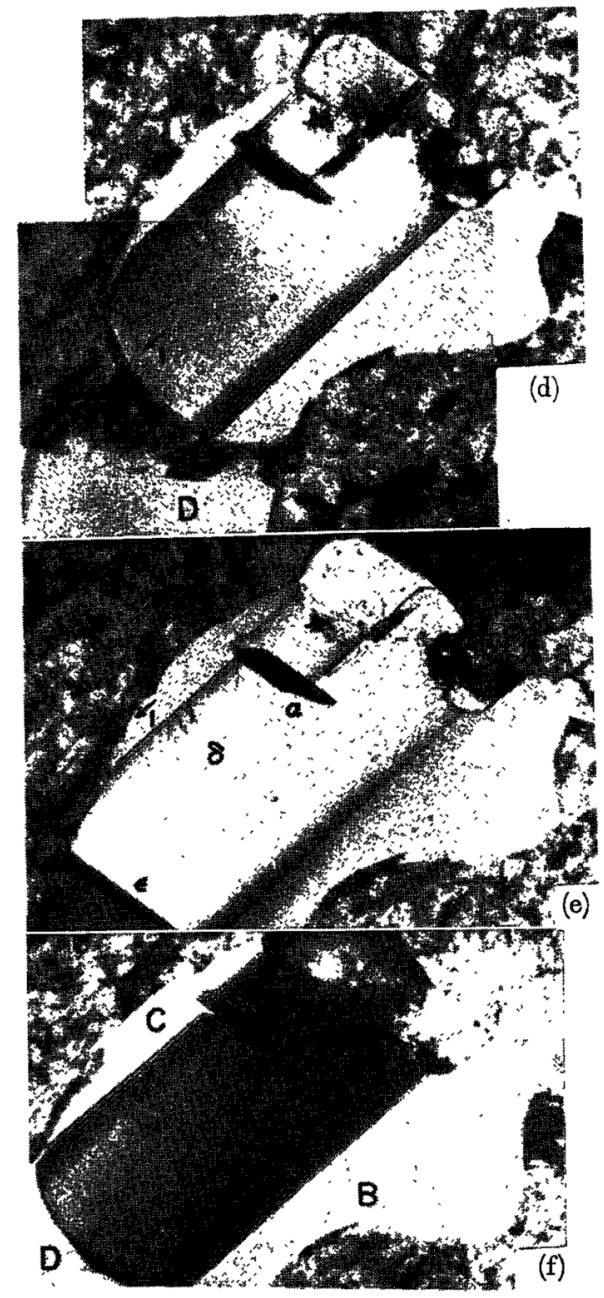

Photo. 1 Successive stages of parallel-sided twin formation in the course of primary recrystallization.

inside the electron microscope. In the first picture (a), a small grain A might have nucleated near the dislocation tangle $\gamma$, because no perceptible change in the tangled region was detected during the growth that followed. Besides the migration of the high-angle boundary to the direction 1, the grain A forms a twin crystal C in the neighboring matrix as shown in Photo. (c). After a certain incubation period, the original high-angle boundary was replaced by a coherent twin boundary and the other high-angle boundary. In order for this situation to occur, the twinning plane must be nearly parallel to the highangle boundary in motion. Several line images were observed on the coherent twin boundary between the grains $\mathrm{A}$ and $\mathrm{C}$, and the lines disappeared gradually with further annealing, as seen in the series of micrographs in Photo. 1. A tiny grain $\mathrm{B}$ growing in the direction 2 is also in a twin relation with the grain $A$ and is supposed to have been formed by the same mechanism as grain $\mathrm{C}$.

(6) J. E. Burke : Trans. Met. Soc. AIME, 188 (1950), 1324.

(7) W. Bollman : J. Inst. Metals, 87 (1958), 439.

(8) S. Horiuchi, T. Okubo and I. Gokyu :Trans. JIM, 9(1968), 194. grew no more except along the length of the coherent boundaries. The increase of the twin width as reported by Dash and Brown ${ }^{(9)}$ has not been observed in the present experiment.

Stacking faults $\beta$ and $\delta$ or thin twins $\alpha$ were also formed nearly perpendicular to the high-angle boundary during the migration. It is demonstrated in the photographs that these faults remain still even after the original boundary is replaced by the coherent boundary. The detailed description of them will be given in the following section. Some of them, $\alpha$ and $\delta$, shrank or disappeared in the latter stage of grain growth.

\section{Stacking faults and thin twins}

Another feature of the observed phenomena was the frequent formation of stacking faults or thin twins following the migration of the high-angle grain boundaries, as shown in Photo. 2. In this situation, the specimen was so oriented that a tetrahedral plane (111), along

(9) S. Dash and N. Brown : Acta Met.,11 (1963), 1067. 
which the stacking faults or twins could be formed, made a large angle with the migrating boundary, in contrast to the parallel-sided twins. The defects $\alpha, \zeta$ or $\eta$ are found to be single layers of stacking fault, whereas others are thin twins, as evidenced from a set of fault fringes and the sequence of the fault layers. When a part of the migrating boundary reached a region, i. e. the middle of Photo. 2(a), stacking faults began to form. Accompanying the boundary migration the partial dislocations moved in a family of the tetrahedral planes, so that the stacking faults extended as seen in the photographs. During the boundary migration each stacking fault of ten developed into a small packet of stacking faults, i. e. thin twins $\beta$, $\delta, \varepsilon$ and $\theta$, etc., by forming successive stacking faults layer by layer.

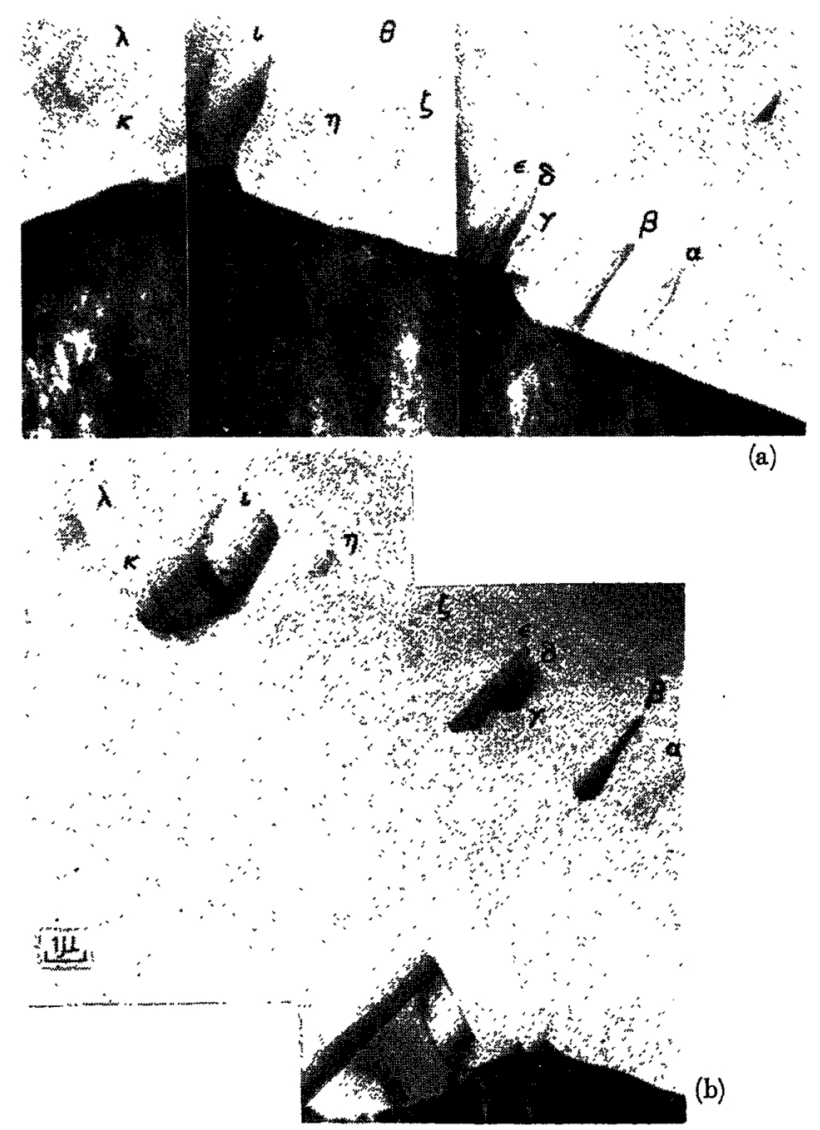

Photo. 2 Formation of stacking faults and thin twins according to the migration of the recrystallized grain boundary. (a) shows the earlier stage and (b) the later stage on the same domain.

The dislocation mechanism accounting for the thickening of the faults is considered to be based on the pole mechanism, as will be accepted from a dislocation array on a coherent boundary of the twin $\iota$ in Photo. 2 (b). It should be noted that the whole defects belong to one family of the tetrahedral plane except the fault $\zeta$. This suggests that the cause of the faults are directly connected with the local condition of the site in question, including the dislocation arrangement in the matrix, the orientation relation across the boundary and boundary structure.

On further annealing, the faults were detached from the grain boundary and left in the recrystallized grain as seen in Photo. 2 (b). Afterwards some of them shrank longitudinally along the coherent plane by rearranging the (partial) dislocations into arrays on a low index plane, as seen in $\zeta, \eta, \kappa$ and $\lambda$, or disappeared sometimes leaving debris which were considered to be partial dislocations, as in $\gamma$ or $\theta$. After being detached from the boundary, the thickening of these thin twins have never occured. These kinds of thin faults were observed also in the specimens that had been thinned after recrystallization in the bulk state as Dash and Brown have done. Accordingly the formation mode of these faults cannot be ascribed to the effect of the specimen thickness, but it is a substantial way of forming the annealing twins in the high purity nickel, and is believed to be valid in other fcc metals and alloys, too.

\section{Discussion}

Several theories have been proposed to explain the formation mechanism of the annealing twins. It was pointed out by Fullman and Fisher that the formation of annealing twins during the grain growth and the secondary recrystallization originates in the reduction of the net grain boundary energy ${ }^{(10)(11)}$. Burke advanced a growth fault hypothesis, which states that during the primary recrystallization an annealing twin forms if the migrating grain boundary corresponds approximately to a tetrahedral plane of the growing grain at the point where the twinning accident occurs $^{(6)}$. Burgers suggested a somewhat different mechanism relating, to a certain extent, to the growth fault hypothesis; the growth of a stimulated crystal takes place when a growing crystal encounters the nucleus, which is supposed to be a lattice region in the matrix and to be oriented approximately by a twin relation to the growing crystal ${ }^{(12)}$. On such an encounter, the decrease in dislocation density at the place of contact due to the annihilation of dislocations might cause a release of strain energy in the contacted lattice region, which thus could become an active nucleus. Moreover, a few works on the direct observation by transmission electron microscopy have been carried out to investigate how annealing twins nucleate and grow. In particular, Dash and Brown, from their observation, deduced a conclusion that a nucleus for an annealing twin is a layer or a thin packet of stacking fault ${ }^{(9)}$.

Now let us discuss the correlation of the present work with these previous investigations. Among possible ideas the following seems to be most adequate. During the growth of a recrystallized grain, the boundary will encounter various lattice regions polygonizing to some extent. Among these regions there seem to be special ones oriented close to a twin relation, as a grain $A$ in Fig. 1. When the migrating boundary touches such a region, as in (a) of Photo. 1, the interface between the region and the recrystallized grain is expected to transform into a coherent twin plane, superposed by a subboundary in general, by the annihilation or rearrangement

(10) R. L. Fullman and J. C. P. Fisher : J. Appl. Phys., 22 (1951), 1350.

(11) R. L. Fullman : J. Appl. Phys, 22 (1951), 456.

(12) W. G. Burgers : Nature, 157 (1946), 76 ; Proc. Acad. Sci. Amsterdam, 50 (1947), 858; Physica, 15 (1949), 1437. 
of dislocations in the high-angle boundary. A line array on the coherent boundary of the grain $\mathrm{C}$ in Photo. $I$ (a) and (b) is considered to be the dislocations based on the subboundary.
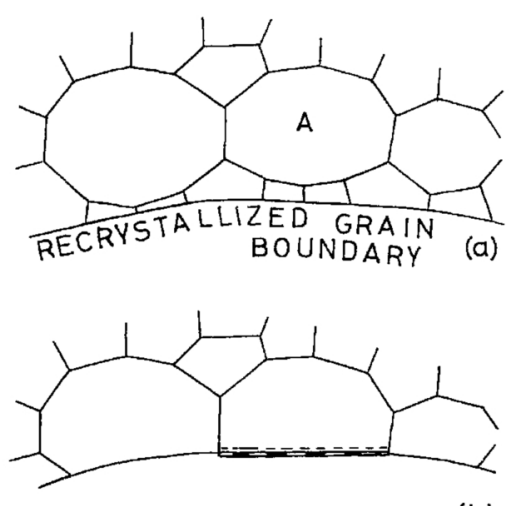

(b)
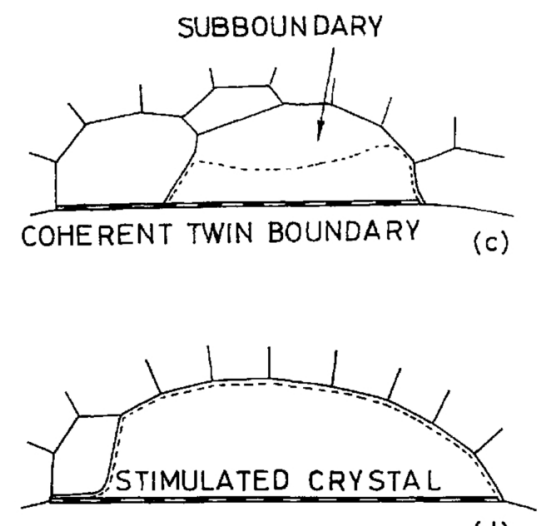

(d)

Fig. 1 Schematic drawings on the formation of a nucleus of parallel-sided twin. A grain A in the matrix is supposed to be of near-twin orientation with the recrystallized grain. The surrounding boundary of grain A becomes energetically unstable owing to the change in boundary structure, resulting in the formation of an extended twin grain.

According as the annihilation of dislocations proceeds, the equilibrium configuration of dislocations near the boundary will be destroyed, and as shown schematically in Fig. 1, the subboundary then leaves from the coherent plane, traversing the grain and being combined with the boundary on the opposite side, possibly increasing the misfit angle across the boundary. The subboundary decomposition similar to that described here has been found in other metals in the course of subgrain growth ${ }^{(13)(14)}$. In the present case, the decomposition of the high-angle boundary into two boundaries, i. e. one coherent boundary and other subboundary, is very probable if the misfit angle of the original boundary is near the energy cusp point of a twin. In this situation it is not always necessary for the migrating boundary to encounter the exactly twin-oriented regions, but the coherent twin boundary can be formed unless the region is far deviated from the twin orientation, i. e. more than few degrees. The occurrence of such parallel-sided twins in the observing crystals was different from place to place, suggesting that the twin

(13) H. Fujita : J. Phys. Soc. Japan, 26(1969), 1437.

(14) E. Furubayashi : Trans. Iron Steel Inst. Japan, 9 (1969), 222. nuclei are stimulated regions with near twin orientation and the frequency of the nuclei formation depends strongly on the local deformation texture.

In order to form such a stimulated grain that can grow as a nucleus of the twin orientation, an incubation period is surely required. After the incubation, an immediate appearance of fringes could be recognized due to the newly formed coherent twin boundary. Then the nucleus grows and the initial vague interface turns to a sharp high-angle houndary.

Besides the above mentioned consideration based on the stimulation theory, the growth fault hypothesis seems to acocunt for the formation of the parallel-sided twins, since these twins always form when the migrating boundary coincides with a tetrahedral plane.

On the other hand, the appearance of a small packet of stacking faults or thin twins in the present high purity nickel was quite similar to that in a $\mathrm{Ni}-\mathrm{Fe}$ alloy by Dash and Brown ${ }^{(9)}$. In contrast to their interpretation however, these faults did not emanate from the grain boundary into the recrystallized grainby a glide process, but it is formed by the following ; when migrating boundary reached a certain zone in the deformed matrix, stacking faults frequently started to grow at the grain boundary and extended gradually along with the movement of the boundary, without growing into a recrystallized grain. Namely the growth of the faults occurs only when these faults are connected with the migrating high-angle boundary. In the growth of the faults, however, the non-coherent as well as the coherent twin boundary must be created in the grain. The movement of the grain boundary is therefore strongly hindered because of the extra energy consumption, and finally these faults are detached from the migrating boundary, being left inside the recrystallized grain. After detached, the stacking faults or thin twins contract longitudinally by the rearrangement of the non-coherent boundaries. They were also observed to disappear by attractive glide of the partial dislocation walls to reduce the length of the stacking faults of high energy, if the conditions are permitted.

Meanwhile, a question arises whether these thin twins can grow to a considerable size. There is no definite evidence which supports the parallel-sided twins to originate from the thin twins, although some possibilities cannot be excluded, e.g. such as the coalescence mechanism by Burke ${ }^{(6)}$. Even if a certain annealing twins would have grown by this mechanism, the fraction for the total amount of twins might be minor.

It should also be noted that in both cases the dislocation structures in the vicinity of the grains boundary is very important for the nucleation of the annealing twins. The association of the partial dislocations with the noncoherent boundary in the annealing twins have been discussed by Votava et al. ${ }^{(15)}$ or Dash and Brown ${ }^{(9)}$. Both groups suggested experimentally or theoretically that all of the three possible $a / 6<112>$ type partial dislocations on one twinning plane contribute equally to the formation of thê annealing twins. There remain some doubts,

(15) E. Votava and A. Berghezan : Acta Met., 7 (1959), 392. 
however, whether the partials are introduced while forming the twins; instead, they seem to result from the rearrangement of the dislocations on the coherent boundary owing to the energy reduction in the system. In the present case of thin twin formation, the stacking fault fringes which suggest the operation of the pole mechanism were often observed as shown in Photo. 2 (b). If this is a reality, the shear strain is continuously created with the boundary migration when thin twins are produced by the successive pile-up process of stacking fault. The driving force for this process might be the internal stress accumulated in local regions in the deformed matrix where stacking faults or thin twins are frequently generated. This idea is also supported by the observation that the formation of the faults is concentrated in a special region, and further that the family of the twins in the region is mostly of one kind. These faults are energetically unstable because non-coherent boundaries are composed of the dislocations with one Burgers vector as in the case of deformation twins. Hence, once the faults are detached from the boundary, they shrink instantly or disappear as far as the partial dislocations are not pinned down to the foil surface or other dislocations. These processes conform fairly well to the observed results. However, this is still somewhat a matter of speculation, and the exact dislocation mechanism which actually operates in the nucleation stage of the annealing twins will remain unresolved.

\section{Summary}

Recrystallization behavior of $96 \%$ rolled high-purity nickel sheets has been examined at elevated temperatures with a high voltage electron microscope of $500 \mathrm{kV}$. The results obtained are summarized as follows.

(1) The recrystallization temperature depends significantly upon the specimen thickness; $500^{\circ} \mathrm{C}$ and $350^{\circ} \mathrm{C}$ for thin specimens observable at $100 \mathrm{kV}$ and $500 \mathrm{kV}$, respectively, and $250^{\circ} \mathrm{C}$ for bulk specimens. This result suggests that one can observe with little difficulty the phenomena, as in the case of those in the bulk state, by using a $500 \mathrm{kV}$ electron microscope.

(2) During the migration of high-angle boundaries of primary recrystallized grains, two kinds of annealing twins, i. e. parellel-sided and thin twins, are observed to form at the migrating boundaries. The former is found to be the most important morphology of the annealing twins in nickel. In either case the twinning plane in a region mostly belongs to a same family, indicating that the occurrence of the annealing twins strongly depends on the local orientation relation across the recrystallized grain boundary.

(3) A mechanism which conforms to the 'stimulation' theory by Burgers has been considered as a most plausible explanation for the phenomena observed in forming parallel-sided twins; in the mechanism proposed, a certain region nearly twin-related to the growing grain is assumed to exist and further the original boundary is postulated to decompose into a coherent and another small angle boundary.

(4) Thin twins are found to result from the thickening of stacking faults through successive layering process during dragging of faults by the migrating boundary. From the evidences obtained so far, the thickening is supposed to be due to the pole mechanism, similarly to the deformation twins. The driving force for the growth of these twins is the reduction of the internal strain energy affected across the migrating boundary.

\section{Acknowledgments}

The authors wish to express their sincere thanks to Prof. U. Hashimoto and Prof. G. Chaudron for their providing an opportunity to study the recrystallization of high-purity nickel, from April of 1968 to March of 1969, under a close cooperation between Japanese and French scientists. They are also very grateful to Prof. H.Fujita, Dr. S. Kajiwara and Mr. Y. Kawasaki for their stimulating and fruitful discussions, and to Mr. T. Kikuchi for his assistance in the microscope operation. 